\title{
COOPERATIVE AND ONLINE LEARNING IN SIGNAL PROCESSING
}

\author{
Amir Asif and John Nesbit \\ Technical University of British Colombia \\ Surrey, BC V3R 7P8, Canada \\ Email: \{asif, nesbit\}@techbc.ca
}

\begin{abstract}
The paper describes how the Technical University of British Columbia (TechBC) is using online and cooperative learning to improve the quality of education offered to its students. To explain how web technology is being used to enhance learning, we focus on a sophomore level course in signal processing. Students study presentations delivered on the Web, discuss problems using an asynchronous conferencing system, and meet on campus once per week for a cooperative laboratory session. Comparative research indicates that introducing cooperative learning in university courses results in higher student achievement and more positive attitudes toward the subject and teaching methodology. Delivery of materials and learning activities via the Web provides greater access and flexibility for students. The students used a course management system (CMS) to study the online portion of the course.
\end{abstract}

\section{INTRODUCTION}

Advances in the educational technology made possible by the World Wide Web (WWW) provide an opportunity to re-think how learning can be supported in the university programs. A common first response to new technology is to use it to reproduce or complement traditional methods, as occurs when lecture notes are made available on a course web site or when lectures are delivered at a distance using satellite-based video conferencing. Effective use of the WWW for teaching and learning, however, requires careful course design and development.

The Technical University of British Columbia (TechBC), Canada's newest public university, welcomed its initial cohort of firstyear students in August, 1999 with a curricular emphasis on Information Technology (Electrical and Computer Engineering, and Computer Sciences), Management and Technology, and Interactive Arts. The development of a strategy for the use of innovative pedagogy and educational technology began before, and extended throughout the planning and development of the first year curriculum. In the early institutional planning for educational technology and learning, four standards emerged:

- Emphasize collaborative learning with classes organized in teams - On average, half of the learning contact time occurs online.

- Most courses integrate online and face-to-face learning.

- All courses include a substantial web-based component.

Hundreds of research studies on cooperative learning [3] have established that the introduction of structured, team-based activities has substantial positive impact. Making course material available on the WWW allows flexibility and round the clock access to the learners. In particular, the amount of time students need to spend on the campus is reduced. Integrating asynchronous online activities with face-to-face interaction enables application of the multimedia features to improve pedagogy without eliminating the realtime social interaction.

It was apparent that achieving these standards for the first set of courses and the small initial cohort of students would be easier than maintaining them as student enrollment and course offerings grew rapidly. To enable scalability it was decided to design a relatively small set of course delivery models consisting of appropriate combinations of learning methods and technologies. Each course is assigned to a delivery model so that the technical and pedagogical support and planning can be organized around the delivery models rather than the unique requirements of each course. Having each of the delivery models represented in the initial set of course offerings also ensured that a variety of approaches would be explored and compared at the outset.

This paper describes how a new course delivery model developed at TechBC was applied to Signal Processing and Communications (SPC), an introductory signal processing course. In section 2 we present some of the unique features of the course management system (CMS), an interface used by the students to access the curriculum. Section 3 describes a variety of models used at TechBC to deliver courses. Sections 4 and 5 explain how a signal processing course was developed using the CMS and the online environment. In section 6 we conclude the paper.

\section{TECHBC COURSE MANAGEMENT SYSTEM}

The support provided by the course management system (CMS) is crucial to the successful implementation of the course delivery models. After reviewing several commercially available systems it was decided to build a web-based CMS with core functionality written in XML/RPC, Dynamic HTML, and JavaScript. Specialized functions were provided by different commercial products. For example, we have used FirstClass and WebBoard for online conferencing and QuestionMark's Perception for online tests and quizzes. To promote learner engagement, features of the TechBC CMS were designed to provide convenient access to course content and interaction with instructors and other learners. The notable features of the CMS include:

1. An entry page designed to support community building by providing general announcements, Java-based text chat serving as a virtual cafe or hallway, voting on community issues, and links to online conferences dealing with a variety of student concerns.

2. A personal page (Fig. 1) providing links to courses, emails, a calendar, announcements, and personal file space on a server. 


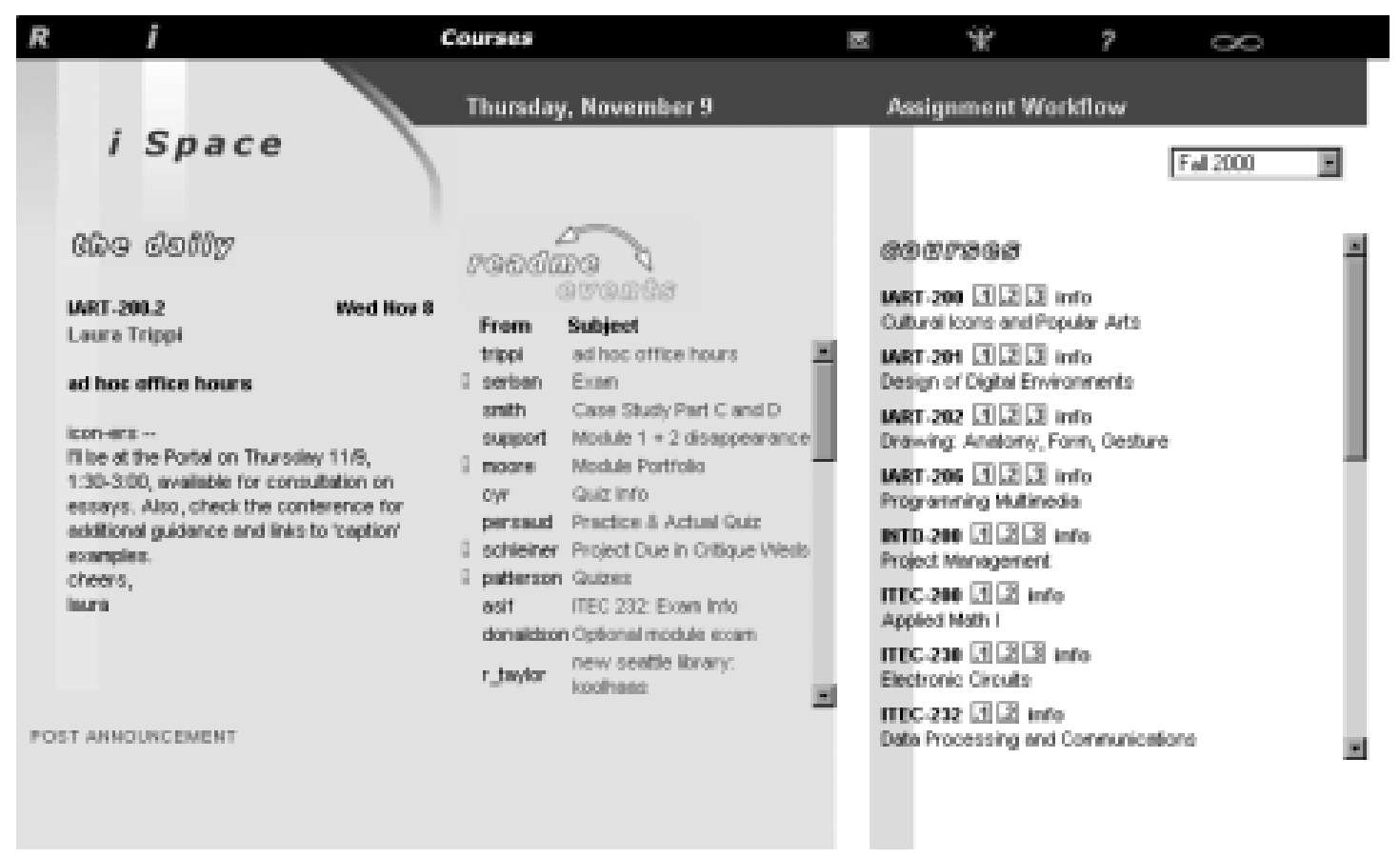

Fig.1: The iSpace provides a calendar, announcements, and links to courses.

3. A syllabus page (Fig. 2) providing links to learning objectives, presentations, online conferences, and assignments and exams.

4. A teams page for each course listing all class members by team. The teams page also provides access to team conferences.

5. A navigation bar (shown at the top in Fig. 1) available throughout the CMS.

The course developers, faculty and teaching staff, were provided with preformatted web page templates specific to the delivery model for which they were working. They also received support from educational technology staff for the creation and licensing of interactive and multimedia elements. Many of the web page templates included interactive components referred to as learning objects. For example, a template in one delivery model included links to prestudy and post-study quizzes. Similarly, other courses provided Java applets, Quicktime movies, and other animations.

Students access the course management system from the oncampus labs and from home. TechBC requires that they have access to the Internet through a 3rd party ISP at a minimum capacity of $56 \mathrm{kbps}$. In fact, learner's home subscriptions and modem speeds range from $28.8 \mathrm{kbps}$ dial-up to cable modem and ADSL. TechBC is located in the lower mainland of British Columbia, a region in which both ADSL and cable subscriptions are available for under \$30 USD per month.

Despite developers' attempts to optimize streaming video for $56 \mathrm{kbps}$, anecdotal reports from TechBC learners indicated that broadband access may be necessary to enable effective home use of, for example, the streaming video presentations. Some learners with dial-in connections report having to come to on-campus labs to effectively use streaming video. As a result, there has been rapid growth in students' subscriptions to broadband Internet services (ADSL and cable) since September, 1999 (Fig. 3). By Fall 2000, over $80 \%$ of the TechBC students had broadband subscriptions.

\section{DELIVERY MODELS}

A course delivery model is a combination of logistical, technologi$\mathrm{cal}$, and pedagogical features. Each model has implications for the scheduling of classrooms and labs, division of roles among teaching staff, course development time, demographic profile of learners, learners' hardware and network requirements, fit to curricula, and so on. Our design of logistical and technological components of the delivery models was influenced by, among others, Bates [1] who discussed access, cost, teaching and learning, interactivity and user-friendliness, and organizational issues as key criteria for making decisions about educational technology and media.

The pedagogical components of the delivery models were influenced by theory, research and practice in asynchronous learning networks [2], cooperative learning [3] and instructional design [4]. The models were designed to shift one-way delivery of information out of lectures toward print or web media, or better yet, to replace one-way delivery with interactive approaches. Among the methods adopted were the use of explicit learning objectives, team-based learning in both face-to-face and online settings, moderated discussions, primary trait analysis for assessment, and interactive simulations. Curricular needs also influenced the design of the models. The following sections summarize the five delivery models currently being used at TechBC.

Presentational Cooperative (PC) Model: The PC model is designed for a section size of 48 learners. It requires study of a web presentation for about 1.5 hours every week prior coming to the face-to-face session which lasts another 1.5 hours each week. The web presentation contains multimedia and other interactive elements. During weekly face-to-face sessions students work in teams of 4 to 6 on structured cooperative learning tasks. An asynchronous online conference called Q\&A board, is provided for students to ask questions. Some courses add open lab sessions where students receive help and work on assignments or projects. 


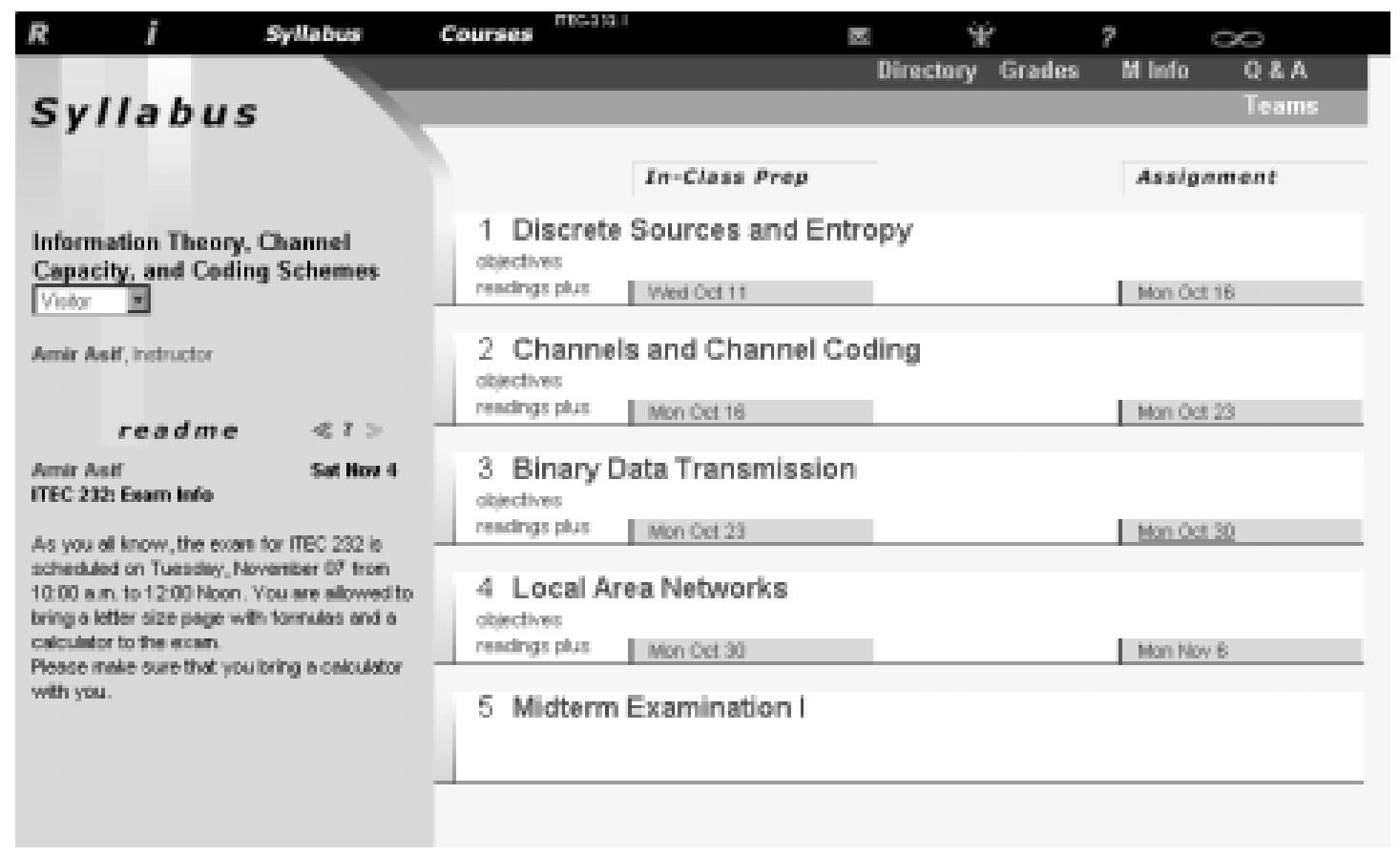

Fig. 2: Syllabus page for the first module of Data Processing and Communications.

Studio Lab (SL) Model: The recommended section size for the SL model is 24 learners. This model bears some similarity to the PC model, but differs in the duration of required on-campus sessions ( 3 hours), and in the learning activities carried out in those sessions. Studio sessions are held in a computer lab, and the learning activities tend to be project based. Some courses add optional lab sessions where students come to receive help on projects.

Computer-Mediated Classroom (CMC) Model: The preferred section size for CMC is 24 learners. The CMC model is built on the tradition of asynchronous learning networks [2] and emphasizes online conferencing. Students are pre-assigned to teams of six, with each team carrying out some of its work within its own conference. Every week, a section of 24 learners takes up a new topic in an online conference. The learners are expected to spend about 3 hours every week on online conferencing. In addition, they do individual assignments. A web-based presentation is also provided. The CMC model imposes a relatively low development cost, but the need to moderate and manage conferences may result in higher delivery costs.

Flexible Study (FS) Model: Derived from the distance learning tradition, this is a self-paced model with no requirement to attend face-to-face sessions. The section size is therefore unlimited. The learners study the web presentation for an average of 3 hours per week. An asynchronous online conference is provided for students to ask questions. To compensate for the absence of scheduled on-campus sessions, web-based conferences and peer contact information are provided to support the formation of informal study groups. The flexible study model demands the production or acquisition of high quality web-based and multimedia resources. Compared to other models described here, it imposes the greatest development costs but the least delivery costs. There are frequent self-diagnostic quizzes and less frequent formal examinations.

\section{SIGNAL PROCESSING AND COMMUNICATIONS}

Signal Processing and Communications (SPC) is a second year course consisting of three modules ${ }^{1}$ that are compulsory for all IT learners at TechBC. The intent of the second year courses within the IT program area is to provide a background on various IT concentrations offered in the third year, where the learners make a selection and specialize in one concentration. The four concentrations presently being offered in IT are: Telecommunications Engineering, Computer Systems Engineering, Software Engineering, and Software Systems. SPC is a core course for the telecommunications engineering concentration and an important applied course for the other three concentrations. The delivery model selected for the course is PC because SPC will eventually enroll a relatively large number of students who are expected to benefit from carrying out laboratory activities in small groups. In addition, the PC model affords smaller class size and more discovery through team discussions.

The text selected for the course is the Oppenheim text on Signals and Systems [5]. We also found an excellent laboratory text compiled by V. Stonick and K. Bradley [6] that is being used to simulate Matlab projects to augment the theory and concepts from the text. Below we describe how the course is structured.

Prior Preparation: Before coming to the face-to-face sessions, learners are expected to review the related material from the text. To facilitate learning, an online component is designed for the course that includes a web presentation to supplement the text and explain difficult concepts, a description of the class activities for the face-to-face session, and assignments to be solved individually by the learners. Learning objects, for example, Java applets and other animations accompany the online explanation for important concepts and difficult derivations.

\footnotetext{
${ }^{1} \mathrm{~A}$ module at TechBC is equivalent to a credit at many traditional university. The three modules are independent and are graded separately.
} 
To ensure that the learners are coming prepared to the faceto-face sessions, we ask them to write a short quiz. Right now the quiz is being written during the face-to-face session, but we are implementing a web-based quiz facility that allows round the clock access. It automatically records the performance of each learner using multiple-choice numerical questions. This provides timely data on where the sticky spots are for the learners, which hopefully can be addressed in the coming face-to-face session.

Cooperative Learning: The cooperative laboratory activity designed from [6] is the focus of the face-to-face sessions. The students work in small groups of four. We believe that learning signal processing is best motivated by applications, and thus have included a number of signal processing applications in the activities. The applications include:

-Music Synthesis of Beethoven's Fifth symphony: Students synthesize the audio tones that make up a well-known sequence from Beethoven's Fifth Symphony by using basic mathematical functions like a sinusoidal tone as fundamental building blocks.

-Fourier Sound Synthesis: Students investigate how to use a computer to synthesize audio signals from the Fourier series coefficients. Then similar shaped waveforms having different pitches are generated to create interesting musical sounds.

-Speech Modeling and Prediction: Students learn how to generate digitally synthesized speech by using a difference equation model for digital speech.

-Audio Tape Restoration: Students restore an audio tape that has been corrupted by an annoying hiss by designing and simulating analogue and digital Butterworth filters.

- Image Deblurring and Decoding: Students use frequency-domain techniques to analyze the effect of blurring in static images. Using 2D filters, they also reduce blurring present in the images.

-Amplitude modulation: Students analyze, design, synthesize, and simulate an amplitude modulation radio system.

The only hardware needed is a Pentium with a sound card and a head-phone microphone. The software needed is Matlab from MathWorks. Students review the instructions of the activities, perform part of the activity in the face-to-face session, and complete the rest in their spare time.

Feedback: To answer queries or provide feedback to the learners, we have designed a moderated question and answer (Q\&A) board used by the whole class. The instructor monitors the Q\&A board and answers the important concerns and questions. Each group also has a separate asynchronous conference that may be used to coordinate group activities. Feedback may also be provided through an open lab where the learners access the lab facilities. We also maintain a list of frequently asked questions (FAQs), which the learners can explore if and when desired.

Assignments: Students access the assignments and submit their individual solutions through the WWW. The solutions for the assignments are also provided online. Since students work on a variety of exercises, it is important to give them a single place to turn for a summary of their scores and current standings relative to that of others in the class. The facility also allows students to review their performance in assignments, examinations, and quizzes.

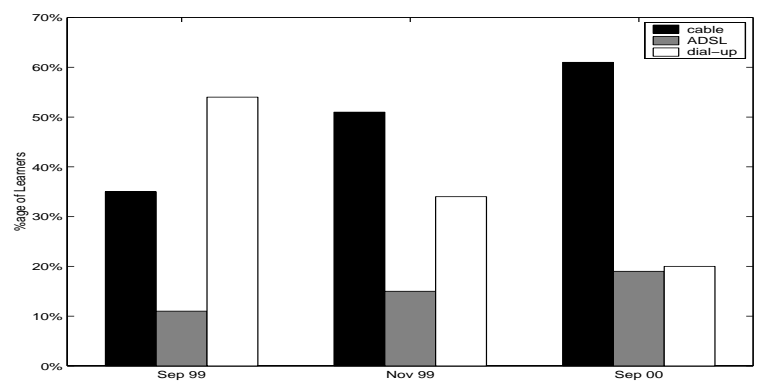

Fig. 3: Learners with Cable, ADSL, and dial-up connections.

\section{DELIVERY OF SPC}

SPC will be delivered for the first time in the Winter semester of the year 2001. We will then be able to compare the effectiveness of the newly designed course with the results from a number of departments across Canada and United States. In the first year curriculum completed by the TechBC learners in May 2000, results of online learning have been highly encouraging. The cooperative team-based approach had a positive effect in the performance of the low-achieving students. At the same time, the students appreciated round the clock accessibility to the course material. Compared to standard lecturing, the instructors also found the delivery effective, manageable and less intensive.

\section{SUMMARY}

In this paper, we describe how TechBC is using advanced educational technologies to enhance the quality of education offered to its students. In a signal processing course, for example, we replaced lectures with web presentations containing multimedia and interactive elements. The students review the presentations before attending the face-to-face sessions where they work in teams on cooperative learning tasks or projects that are applications of the important concepts covered in the presentations. Q\&A boards are available for asynchronous conferences of the team members. An online facility allows students to submit assignments and project reports, and receive feedback. An online gradebook is available to allow students to review their marks and overall performance relative to others in the class. A web-based quiz facility is being implemented that will provide timely data on where deficiencies lie among learners, which can be addressed in face-to-face sessions.

\section{REFERENCES}

[1] A. W. Bates, "Technology: Open Learning and Distance Education," UK: Routledge, London, 1995.

[2] L. Harasim, S. R. Hiltz, L. Teles, and M. Turoff, "Learning Networks: A field Guide to Teaching and Learning Online," MA: MIT Academic Press, Cambridge, 1995.

[3] B. J. Millis and P. J. Cottell, "Cooperative Learning for Higher Education Faculty," AZ: Oryx, Phoenix, 1998.

[4] R. M. Diamond, "Designing and Assessing Courses and Curricula," SF: Jossey-Bass, 2nd ed., 1998.

[5] A. V. Oppenheim, A. S. Willsky, and S. H. Nawab, "Signals and Systems," NJ: Prentice Hall Publishers, 2nd ed., 1983.

[6] V. Stonick, and S. H. Nawab, "Labs for Signals and Systems using Matlab," NY: PWS Publishing Co., 1st ed., 1996. 